\title{
Mehr Sicherheit mit zentraler Bankenaufsicht
}

\section{David Gmür}

\section{Relevanz}

Die Wirtschaft braucht Kredit, und die Gesellschaft mehr Sicherheit. Die Banken müssen in der Kreditvergabe auf Ertrag und Risiko gleichzeitig achten. Damit die Regulierung effektiven Schutz bieten kann, muss die Bankenaufsicht die Einhaltung der Vorschriften überwachen und frühzeitig das Entstehen übermässiger Risiken aufdecken. In der Bankenunion kann eine zentrale Aufsicht eher für gleich lange Spiesse im europaweiten Wettbewerb der Banken sorgen, kann unabhängiger als nationale Aufsichtsbehörden agieren, und dank besserer Ressourcen auch komplexe Grossbanken wirksam beaufsichtigen. So wird die Kreditvergabe sicherer, indem die Banken angehalten sind, faule und riskante Kredite zügig abzubauen und mehr Kredit auf die produktiveren Unternehmen mit besseren Aussichten lenken.

Christian Keuschnigg und Michael Kogler, Herausgeber.

\section{Quelle}

Altavilla, Carlo, Miguel Boucinha, José-Luis Peydró, und Frank Smets (2020), Banking Supervision, Monetary Policy and Risk-Taking: Big Data Evidence from 15 Credit Registers, CEPR Discussion Paper Nr. 14288.

D. Gmür $(\bowtie)$

Universität St.Gallen, St.Gallen, Schweiz

E-Mail: david.gmuer@student.unisg.ch 
Im November 2014 trat in der Eurozone der einheitliche Bankenaufsichtsmechanismus in Kraft, der ein wesentlicher Pfeiler der Europäischen Bankenunion ist. Seither unterstehen grosse Banken der Eurozone einer einheitlichen überstaatlichen Regulierung und Aufsicht durch die Europäische Zentralbank. Die Regulierung von Banken ist eine komplexe Aufgabe. Wie die Finanzkrise 2008/2009 zeigte, konnte die Regulierung nicht verhindern, dass Banken übermässige Risiken eingingen. Welche organisatorischen und institutionellen Reformen können dazu beitragen, die Stabilität des Finanzsystems zu erhöhen? Wichtig ist, ob die Banken durch nationale oder überstaatliche Aufsichtsbehörden überwacht werden.

Während nationale Aufsichtsbehörden besseren Zugang zu genaueren Informationen über den lokalen Bankensektor haben, kann eine überstaatliche Behörde über mehr Ressourcen wie beispielsweise qualifizierte MitarbeiterInnen verfügen. Auch die Anreize können sich je nach Regulierungsebene unterscheiden. Angesichts des häufigen Personalwechsels zwischen lokalen Behörden und Banken sowie starkem Lobbying sind nationale Behörden anfälliger für Interessenkonflikte und tendenziell nachsichtiger in der Aufsicht. Dagegen kann eine überstaatliche Behörde unabhängiger agieren.

Stimmt das? Welche Auswirkungen hatte konkret der Systemwechsel von nationaler zu supranationaler Bankenaufsicht in der Eurozone auf die Kreditvergabe der Banken und die Stabilität des Finanzsystems? Carlo Altavilla, Miguel Boucinha, Jose-Luis Peydro und Frank Smets von der Europäischen Zentralbank sowie der Universität Pompeu Fabra in Barcelona gehen dieser Frage nach. Sie untersuchen Daten aus 15 Europäischen Kreditregistern mit rund 280 Mio. Beobachtungen im Zeitraum von Juni 2012 bis Dezember 2017. In ihrer Analyse nutzen sie die Tatsache, dass nicht alle, sondern nur die bedeutenden Banken unter die neue supranationale Aufsicht fallen. Zum Vergleich berücksichtigen sie auch das Verhalten von Banken ausserhalb der Eurozone, welche nicht davon betroffen waren. Dadurch können sie die geschätzten Effekte tatsächlich dem Systemwechsel zuordnen.

Ihre Schätzungen zeigen, dass die Banken bei überstaatlicher Aufsicht ihre Kreditvergabe an Firmen mit hohem Kreditrisiko einschränken und stattdessen die Kredite an weniger riskante Firmen ausweiten. Firmen gelten als riskant, wenn sie mit einem grossen Teil ihrer Kredite in Verzug sind. Die zentralisierte Aufsicht senkt demnach das Risiko im Kreditgeschäft der Banken. Die Banken schichten von riskanteren zu weniger riskanten Firmen um, ohne dass das gesamte Kreditvolumen leidet.

In ihrer Analyse unterscheiden die Forscher zwischen Banken in finanziell stabilen und instabilen Mitgliedsstaaten der Eurozone. Italien, Portugal und Spanien gelten als finanziell instabil. Österreich, Belgien, Frankreich, Deutschland, Litauen und die Slowakei zählen als stabile Länder. Der Effekt des System- 
wechsels ist für finanziell instabile Länder stärker ausgeprägt als für stabile. In der instabilen Gruppe reduziert die zentrale Bankenaufsicht die Kreditvergabe von Banken an Firmen mit dem höchsten Kreditrisiko um $43 \%$. In stabilen Ländern beträgt dieser Rückgang immerhin noch $36 \%$. Steigt das Kreditrisiko (um eine Standardabweichung) an, so geht die Kreditvergabe an diese Firmen bei zentraler Aufsicht in den instabilen Ländern um rund acht Prozent und in den stabilen Staaten um fünf Prozent zurück.

Der Systemwechsel zur überstaatlichen Bankenaufsicht der Eurozone reduziert die Kreditvergabe an Firmen mit dem höchsten Kreditrisiko um 43\% in finanziell instabilen und um 36\% in stabilen Mitgliedsstaaten.

Wie ist dieser Effekt zu bewerten? Problematisch wäre es, wenn die riskanteren Firmen gleichzeitig in besonders produktiven Sektoren tätig wären und nun weniger Kredite erhielten. Die Forscher stellen jedoch fest, dass dies nicht der Fall ist. Dies gilt unabhängig davon, ob die Banken in finanziell stabilen oder instabilen Ländern tätig sind. Die supranationale Bankenaufsicht scheint die Kreditvergabe an produktivere Firmen sogar tendenziell zu erhöhen, allerdings ist dieser Effekt nicht statistisch signifikant. Betrachtet man dagegen Kredite gleicher Qualität, dann vergeben die Banken mehr Kredite an produktivere Firmen. So erhalten Firmen in einem Sektor, dessen Arbeitsproduktivität um eine Standardabweichung über dem Mittelwert liegt, bei gegebenem Risiko um fünf Prozent mehr Kredite.

Bei gleichem Kreditrisiko vergeben Banken um 5\% mehr Kredite an Firmen, deren Produktivität um eine Standardabweichung über dem Durchschnitt liegt.

Die einheitliche Bankenaufsicht in der Eurozone trat im November 2014 in Kraft. Dies war jedoch schon seit Oktober 2013 bekannt. Wann genau traten die Änderungen im Risikoverhalten der Banken ein? Abb. 1 zeigt von Mitte 2013 bis 2015, wie die supranationale Aufsicht die Kreditvergabe an Firmen mit dem höchsten Kreditrisiko in finanziell instabilen Mitgliedsstaaten beeinflusst. Es zeigt sich, dass die Banken teilweise schon vorab ihr Verhalten änderten, d. h. der effektive Systemwechsel könnte schon früher stattgefunden haben. Der Effekt ist jedoch erst in der zweiten Jahreshälfte 2014 signifikant. Banken scheinen daher ihr Risikoverhalten tatsächlich erst dann angepasst zu haben, als die neue Aufsicht im November 2014 ihren Betrieb aufnahm.

Die Forscher gingen noch einen Schritt weiter und analysierten das Zusammenspiel des regulatorischen Systemwechsels mit der expansiven Geldpolitik der Europäischen Zentralbank. Ihre Schätzungen zeigen, dass eine 


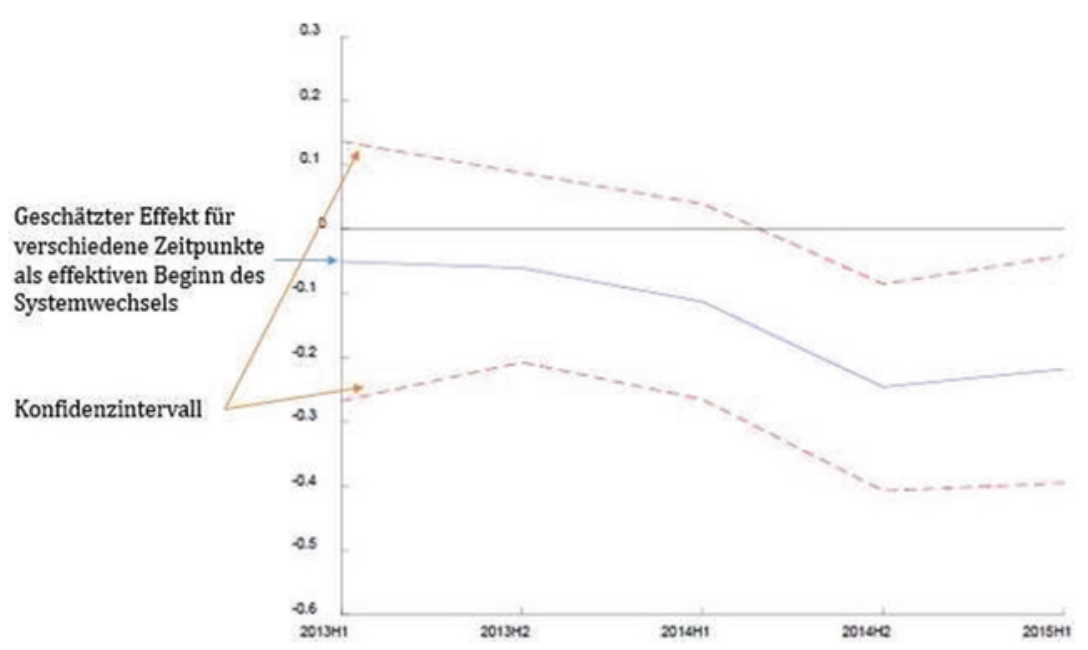

Abb. 1 Effekt der einheitlichen Bankenaufsicht auf die Kreditvergabe an Firmen mit dem höchsten Kreditrisiko. (Quelle: Altavilla et al. 2020)

Lockerung der Geldpolitik dazu beiträgt, dass Banken mehr Risiken eingehen und zusätzliche Kredite an Firmen mit hohem Kreditrisiko vergeben. Der Wechsel zur überstaatlichen Bankenaufsicht hebt diesen Effekt jedoch auf. Die Kreditvergabe an weniger riskante Firmen wird dabei nicht beeinträchtigt.

Eine Lockerung der Geldpolitik führt dazu, dass Banken mehr Kredite an besonders riskante Firmen vergeben. Die Zentralisierung der Bankenaufsicht in der Eurozone kann diesen destabilisierenden Effekt jedoch aufheben.

Weshalb ist die Aufsicht durch eine überstaatliche Behörde die offenbar effektivere? Das Forscherteam stellt dafür zwei Hypothesen auf. Die Anreizhypothese stellt auf die mögliche Veränderung der Anreize der Aufsichtsbehörden ab, die bei zentraler Aufsicht unabhängiger agieren können, während die Kapazitätshypothese die Bedeutung einer qualitativ und quantitativ besseren Ressourcenausstattung der überstaatlichen Behörde betont.

Sie stellen fest, dass die zentralisierte Aufsicht vor allem das Risikoverhalten von sehr grossen Banken mit einer Bilanzsumme von mehr als EUR $500 \mathrm{Mrd}$. stark beeinflusst. Da die Komplexität der Regulierung für jene Banken überproportional zunimmt, ist dies ein Indiz für die Kapazitätshypothese. Für die 
Anreizhypothese finden die Forscher hingegen kaum Hinweise. So ist beispielsweise bei sehr schwachen Banken, welche von einer lokalen Behörde stärker geschützt werden könnten, keine Veränderung des Risikoverhaltens festzustellen. Sie kommen daher zum Schluss, dass die effektivere Regulierung in erster Linie darauf zurückzuführen ist, dass die supranationale Behörde über mehr Mittel und Personal verfügt, um eine effiziente Kontrolle auch in sehr komplexen Fällen zu gewährleisten.

Die effektivere Aufsicht ist vorwiegend darauf zurückzuführen, dass die supranationale Behörde über mehr Mittel und spezialisiertes Personal verfügt.

Die Studie des Forscherteams um José-Luis Peydró zeigt, dass die Wahl der Aufsichtsebene einen wesentlichen Einfluss auf das Risikoverhalten der Banken und somit auf die Stabilität des Finanzsystems haben kann. Im Fall der Eurozone hat der Wechsel von nationaler zu supranationaler Regulierung dazu geführt, dass die Banken ihre Risiken im Kreditgeschäft trotz der lockeren Geldpolitik verringert haben.

Open Access Dieses Kapitel wird unter der Creative Commons Namensnennung 4.0 International Lizenz (http://creativecommons.org/licenses/by/4.0/deed.de) veröffentlicht, welche die Nutzung, Vervielfältigung, Bearbeitung, Verbreitung und Wiedergabe in jeglichem Medium und Format erlaubt, sofern Sie den/die ursprünglichen Autor(en) und die Quelle ordnungsgemäß nennen, einen Link zur Creative Commons Lizenz beifügen und angeben, ob Änderungen vorgenommen wurden.

Die in diesem Kapitel enthaltenen Bilder und sonstiges Drittmaterial unterliegen ebenfalls der genannten Creative Commons Lizenz, sofern sich aus der Abbildungslegende nichts anderes ergibt. Sofern das betreffende Material nicht unter der genannten Creative Commons Lizenz steht und die betreffende Handlung nicht nach gesetzlichen Vorschriften erlaubt ist, ist für die oben aufgeführten Weiterverwendungen des Materials die Einwilligung des jeweiligen Rechteinhabers einzuholen.

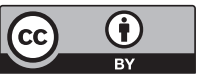

